\title{
The aspherical Cavicchioli-Hegenbarth-Repovš generalized Fibonacci groups
}

\author{
Gerald Williams \\ (Communicated by A. Yu. Olshanskii)
}

\begin{abstract}
The Cavicchioli-Hegenbarth-Repovš generalized Fibonacci groups are defined by the presentations $G_{n}(m, k)=\left\langle x_{1}, \ldots, x_{n} \mid x_{i} x_{i+m}=x_{i+k}(1 \leqslant i \leqslant n)\right\rangle$. These cyclically presented groups generalize Conway's Fibonacci groups and the Sieradski groups. Building on a theorem of Bardakov and Vesnin we classify the aspherical presentations $G_{n}(m, k)$. We determine when $G_{n}(m, k)$ has infinite abelianization and provide sufficient conditions for $G_{n}(m, k)$ to be perfect. We conjecture that these are also necessary conditions. Combined with our asphericity theorem, a proof of this conjecture would imply a classification of the finite CavicchioliHegenbarth-Repovš groups.
\end{abstract}

\section{Introduction}

A group $\Gamma$ is said to be cyclically presented if it has a presentation of the form

$$
\left\langle x_{1}, \ldots, x_{n} \mid w, \eta(w), \ldots, \eta^{n-1}(w)\right\rangle
$$

where $w$ is a word in $X=\left\{x_{1}, \ldots, x_{n}\right\}$ and $\eta$ is an automorphism of the free group $F(X)$ whose action on the generators is given by $\eta\left(x_{i}\right)=x_{i+1}$ (subscripts taken modulo $n)$. Cyclically presented groups have been studied both for algebraic and for geometric reasons.

If $w$ takes the form $w=x_{i} x_{i+m} x_{i+k}^{-1}$ then we obtain a class of groups introduced in [4]. Specifically, the Cavicchioli-Hegenbarth-Repovš generalized Fibonacci groups are the groups defined by the presentations

$$
G_{n}(m, k)=\left\langle x_{1}, \ldots, x_{n} \mid x_{i} x_{i+m}=x_{i+k}(1 \leqslant i \leqslant n)\right\rangle
$$

where all indices are taken modulo $n$ and take their values from the set $\{1, \ldots, n\}$. We shall sometimes refer to $G_{n}(m, k)$ as groups, when we mean the groups defined by the presentations.

The groups $G_{n}(1,2)$ are the Fibonacci groups $F(2, n)$ introduced by Conway [7] (see [18] for a comprehensive survey of such groups); the groups $G_{n}(2,1)$ are the 
Sieradski groups $S(n)$ considered in [15], [19]. The groups $G_{n}(t, 1)$ are the GilbertHowie groups $H(n, t)$ studied in [11].

The asphericity of cyclically presented groups and generalizations of Fibonacci groups have been studied in [1], [6], [11], [14], [16]. In this paper we consider the asphericity of presentations $G_{n}(m, k)$. In [11], Gilbert and Howie give (with certain excluded cases) necessary and sufficient conditions for $G_{n}(t, 1) \cong H(n, t)$ to be aspherical. A presentation $G=A * B$ is aspherical if and only if at least one of the presentations $A, B$ is aspherical (from [1, Lemma 2.1]), and for this reason it is enough to consider only cases where $G_{n}(m, k)$ does not factorize as a free product. Moreover, since the asphericity of presentations $G_{n}(t, 1)$ was considered in [11], we also do not need to consider these cases.

The presentation $G_{n}(m, k)$ is said to be irreducible if $n, m, k$ satisfy

$$
0<m<k<n, \quad(n, m, k)=1,
$$

and is strongly irreducible if it is irreducible and additionally

$$
(n, k)>1, \quad(n, k-m)>1 .
$$

If $G_{n}(m, k)$ is not irreducible then it is either trivial, cyclic, or factorizable into a free product by [1, Lemma 1.2]. If $G_{n}(m, k)$ is irreducible but not strongly irreducible, then it is isomorphic to some Gilbert-Howie group $H(n, t)$ by [1, Lemma 1.3]. (We remark that Edjvet [8] defines irreducibility for an arbitrary cyclically presented group. According to his definition, $G_{n}(m, k)$ is irreducible if and only if $(n, m, k)=1$, and so this is a slightly weaker property than the one used here.)

In [1], Bardakov and Vesnin give sufficient conditions for a strongly irreducible presentation $G_{n}(m, k)$ to be aspherical. In Section 2 we build on this to determine precisely when such presentations are aspherical (Theorem 2). In Section 3 we determine when $G_{n}(m, k)$ has infinite abelianization (Theorem 4) and provide sufficient conditions for $G_{n}(m, k)$ to be perfect (Lemma 5). We conjecture (Conjecture 6) that these conditions are also necessary; using Magma we have verified this for $n \leqslant 200$. Bardakov and Vesnin [1] have asked for a classification of the finite CavicchioliHegenbarth-Repovš groups. We address this question in Section 4 and show that the classification of the finite, strongly irreducible groups $G_{n}(m, k)$ would follow from a proof of Conjecture 6; therefore, we have obtained such a classification for $n \leqslant 200$.

Many of the results in this paper were formulated after performing computational experiments in Magma [2].

\section{Asphericity}

A presentation $P$ is said to be aspherical if $\pi_{2}(P)=0$; the group defined by an aspherical presentation is torsion-free, and hence either trivial or infinite.

For $(n, t) \notin\{(8,3),(9,4),(9,7)\}$ Gilbert and Howie have determined precisely when the presentation $H(n, t)$ is aspherical $([11$, Theorem 3.2]). If $(n, t)=(8,3)$ then a cal- 
culation in Magma shows that $H(n, t)$ defines a finite group of order 295245, and hence $H(8,3)$ is not aspherical.

We shall consider when a strongly irreducible presentation $G_{n}(m, k)$ is aspherical. Bardakov and Vesnin [1] have provided the following sufficient condition.

Theorem 1 ([1]). Let $G_{n}(m, k)$ be strongly irreducible. Then $G_{n}(m, k)$ is aspherical if none of the following conditions are satisfied:

(1) there exists an integer $l \geqslant 1$ such that $n$ divides $l(2 k-m)$ and also $1 / l+(n, k) / n+(n, k-m) / n>1$

(2) $n=k+m$;

(3) $n=2(k-m)$ and $(n, k) \leqslant n / 2$;

(4) $n=2 k$ and $(n, k-m)<n / 2$.

We build on this result to provide necessary and sufficient conditions for $G_{n}(m, k)$ to be aspherical.

Theorem 2. Suppose that $G_{n}(m, k)$ is strongly irreducible. If $(m, k)=1$ and either $n=2 k$ or $n=2(k-m)$ then $G_{n}(m, k)$ is not aspherical. In all other cases $G_{n}(m, k)$ is aspherical.

Before embarking on the proof, we first identify classes of finite cyclic groups among the groups $G_{n}(m, k)$.

Lemma 3. Suppose that $(m, k)=1$ and either (i) $n=2 k$, or (ii) $n=2(k-m)$. Then $G_{n}(m, k) \cong \mathbb{Z}_{s}$ where $s=2^{n / 2}-(-1)^{m+n / 2}$.

Proof. Consider first case (i). Let $P$ be the presentation of $G_{n}(m, k)$ defined as in (1.1). That is, $P$ has generators $x_{1}, \ldots, x_{2 k}$ and relations

$$
x_{i} x_{i+m}=x_{i+k} \quad(1 \leqslant i \leqslant 2 k)
$$

(with subscripts taken modulo $2 k$ ). Setting $i=q-m-k$ in (2.1) we obtain $x_{q-m}=x_{q-m-k} x_{q-k}$; on the other hand, setting $i=q-m$ gives $x_{q-m}=x_{q-m+k} x_{q}^{-1}$. Hence $x_{q-k}=x_{q}^{-1}$ and so we may add the relations

$$
x_{i-k}=x_{i}^{-1} \quad(1 \leqslant i \leqslant 2 k)
$$

to $P$ without changing the group that it defines. Relations (2.1) and (2.2) imply $x_{i} x_{i+m}=x_{i}^{-1}$ and so we may add relations

$$
x_{i+m}=x_{i}^{-2} \quad(1 \leqslant i \leqslant 2 k) .
$$

The relations (2.1) are a consequence of (2.2) and (2.3) and so can be removed. 
Suppose that $m$ is even; then $k$ is odd. By (2.2) we may remove all generators $x_{i}$ where $i$ is odd (and hence also the relations (2.2)). Thus $P$ has generators $x_{2 j}(1 \leqslant j \leqslant m)$ and relations

$$
x_{2 j+m}=x_{2 j}^{-2} \quad(1 \leqslant j \leqslant k),
$$

(from (2.3)). Since $(m, k)=1$ and $m$ is even, $(m / 2, k)=1$, and so for each $j$ with $1 \leqslant j \leqslant k$ there exists a unique $J$ with $1 \leqslant J \leqslant k$ such that $J(m / 2)=j \bmod k$ and hence $J m=2 j \bmod 2 k$. Thus we can write the generators of $P$ as $x_{J m}(1 \leqslant J \leqslant k)$ and the relations (2.4) as

$$
x_{(J+1) m}=x_{J m}^{-2} \quad(1 \leqslant J \leqslant k) .
$$

Then for each $J$ we have

$$
x_{J m}=x_{(J-1) m}^{-2}=x_{(J-2) m}^{(-2)^{2}}=\cdots=x_{m}^{(-2)^{J-1}}=x_{k m}^{(-2)^{J}}
$$

and so we may add relations

$$
x_{J m}=x_{k m}^{(-2)^{J}} \quad(1 \leqslant J \leqslant k) .
$$

The relations (2.5) are a consequence of (2.6) and so we may remove them. Using (2.6) we may remove generators $x_{J m}$ for $1 \leqslant J \leqslant k-1$ together with the corresponding relations, leaving the presentation

$$
P=\left\langle x_{k m} \mid x_{k m}=x_{k m}^{(-2)^{k}}\right\rangle=\left\langle x_{k m} \mid x_{k m}^{(-2)^{k}-1}=1\right\rangle,
$$

and so (since $m$ is even and $k$ is odd) $G_{2 k}(m, k) \cong \mathbb{Z}_{s}$, as required.

Suppose then that $m$ is odd. Then $(m, 2 k)=1$ and so for each $i$ with $1 \leqslant i \leqslant 2 k$ there exists a unique $J$ with $1 \leqslant J \leqslant 2 k$ such that $i=J m$. Thus the generators of $P$ can be written as $x_{J m}(1 \leqslant J \leqslant 2 k)$, the relations $(2.2)$ as

$$
x_{(J+k) m}=x_{J m}^{-1} \quad(1 \leqslant J \leqslant 2 k),
$$

and $(2.3)$ as

$$
\begin{aligned}
x_{(J+1) m} & =x_{J m}^{-2} \quad(1 \leqslant J \leqslant k-1), \\
x_{(k+1) m} & =x_{k m}^{-2}, \\
x_{(J+k+1) m} & =x_{(J+k) m}^{-2} \quad(1 \leqslant J \leqslant k-1), \\
x_{m} & =x_{2 k m}^{-2} .
\end{aligned}
$$

Using (2.7) we can remove generators $x_{(J+k) m}(1 \leqslant J \leqslant k)$ and the relations $(2.7)$. Since $x_{(J+k) m}=x_{J m}^{-1}$ for each $J$ the relations (2.10) become equivalent to (2.8) and so can be removed; relations (2.9) and (2.11) both become

$$
x_{m}=x_{k m}^{2} .
$$


Thus $P$ has generators $x_{J m}(1 \leqslant J \leqslant k)$ and relations $(2.8)$ and (2.12). For each $J$ we therefore obtain

$$
x_{J m}=x_{(J-1) m}^{-2}=x_{(J-2) m}^{(-2)^{2}}=\cdots=x_{(J-(J-1)) m}^{(-2)^{J-1}}=\left(x_{k m}^{2}\right)^{(-2)^{J-1}} .
$$

We can therefore add the relations

$$
x_{J m}=x_{k m}^{-(-2)^{J}} \quad(1 \leqslant J \leqslant k)
$$

The relations (2.8) are a consequence of these, so we may remove them. Using (2.13) we may remove generators $x_{J m}$ for $1 \leqslant J \leqslant k-1$ together with the corresponding relations, leaving the presentation

$$
P=\left\langle x_{k m} \mid x_{k m}=x_{k m}^{-(-2)^{k}}\right\rangle
$$

Since $m$ is odd we have $G_{2 k}(m, k) \cong \mathbb{Z}_{s}$, as required.

Consider then case (ii). We shall use [1, Theorem 1.1] to show that if a number $K$ is coprime to $m$ then the group $G_{2 K}(m, m+K)$ is isomorphic to $G_{2 K}(m, K)$. To this end, let $K^{\prime}=m+K, m^{\prime}=m, r=(2 K, K-m)$. Then $r=(m, K)=1$, and so there exists $J$ with $1 \leqslant J \leqslant 2 K$ such that $J(K-m)=1 \bmod 2 K$. Let $i=1, j=-m J \bmod 2 K$; then $1 \leqslant i \leqslant r, 1 \leqslant j \leqslant 2 K / r$, and

$$
i+j(K-m)=1-m \bmod 2 K, \quad i+j K^{\prime}=1+m^{\prime} \bmod 2 K .
$$

By [1, Theorem 1.1] we have $G_{2 K}(m, m+K) \cong G_{2 K}(m, K)$ as claimed; since also $(m, K)=1$ part (i) of this lemma shows that this is isomorphic to $\mathbb{Z}_{s}$ where $s=2^{K}-(-1)^{K+m}$. Now if $n=2(k-m)$, then setting $K=k-m$ gives that $G_{n}(m, k)=G_{2 K}(m, m+K) \cong \mathbb{Z}_{s}$ where $s=2^{n / 2}-(-1)^{m+n / 2}$, as required.

Proof of Theorem 2. If $(m, k)=1$ and either $n=2 k$ or $n=2(k-m)$ then by Lemma 3 the group $G_{n}(m, k)$ is finite and non-trivial, and so is not aspherical. Suppose then that neither of these possibilities occurs. We shall show that none of the conditions (1), (2), (3), (4) of Theorem 1 holds, and hence $G_{n}(m, k)$ is aspherical.

Condition (1). If $n /(n, k)=2$ then (since $0<k<n$ ) we have $n=2 k$, and so by our assumption, $(m, k)>1$. On the other hand, $(n, m, k)=(2 k, m, k)=(m, k)>1$, a contradiction. If $n /(n, k-m)=2$ then $n=2(k-m)$ and so by our assumption, $(m, k)>1$. On the other hand, $(n, m, k)=(2(k-m), m, k)=(m, k)>1$, a contradiction.

Thus we may assume that $n /(n, k), n /(n, k-m) \geqslant 3$, and so the inequality $1 / l+(n, k) / n+(n, k-m) / n>1$ implies that either (i) $l=1$ or (ii) $l=2$ and $n /(n, k)=p, n /(n, k-m)=q$ where $\{p, q\}=\{3,3\},\{3,4\},\{3,5\}$. 
Suppose that $l=1$. Then $n$ must divide $(2 k-m)$. But $k<n$ implies that $2 k-m<2 n$ and hence $n=2 k-m$. Then $1=(n, m, k)=(2 k-m, m, k)=(m, k)$ and $1<(n, k)=(2 k-m, k)=(m, k)$, a contradiction.

Suppose then that $l=2$. Then $n /(n, k)=p, n /(n, k-m)=q$ imply $\alpha n=p k$, $\beta n=q(k-m)$ for some $\alpha, \beta$ with $(\alpha, p)=1,(\beta, q)=1$. Thus $\beta p k=\alpha q(k-m)$ and hence $\alpha q m=(\alpha q-\beta p) k$. But $m>0$ and $k>0$, so that $\alpha q>\beta p$.

If $p=q=3$ then $\alpha, \beta \in\{1,2\}$ and $3 \alpha>3 \beta$ so $\alpha=2, \beta=1$. That is, $2 n=3 k$ and $n=3(k-m)$ and so $k=2 m, n=3 m$. Then $(n, m, k)=m=(n, k-m)$. But $(n, m, k)=1,(n, k-m)>1$, and we have a contradiction. If $p=3$ and $q=4$ then $\alpha \in\{1,2\}, \beta \in\{1,3\}$ and $4 \alpha>3 \beta$, so that $(\alpha, \beta)=(1,1)$ or $(2,1)$. If $(\alpha, \beta)=(1,1)$ then $n=3 k, n=4(k-m)$, so that $k=4 m, n=12 m$, and then $(n, k, m)=1$ implies $m=1$, and so $k=4, n=12$. But then $n \times l(2 k-m)$, and so condition (1) does not hold. If $(\alpha, \beta)=(2,1)$ then $2 n=3 k, n=4(k-m)$. Then $5 k=8 m$ so $5 \mid m$; let $m=5 M$, say. Then $k=8 M, n=12 M$, and $(n, m, k)=1$ implies $M=1$. Hence $m=5, k=8, n=12$ and so $n \nmid l(2 k-m)$.

Similar arguments show that if $(p, q)=(4,3)$ then the tuple $(n, m, k)$ is $(12,5,9)$ or $(12,1,9)$; if $(p, q)=(3,5)$ then $(n, m, k)$ is one of $(15,2,5),(15,7,10),(15,4,10)$ or $(15,1,10)$; if $(p, q)=(5,3)$ then $(n, m, k)$ is one of $(15,1,6),(15,4,9),(15,7,12)$ or $(15,2,12)$. In each case $n \times l(2 k-m)$.

Condition (2). Strong irreducibility implies

$$
(n, m, k)=1, \quad(n, k)>1 .
$$

But $n=k+m$ implies $(n, m, k)=(n, k)$, a contradiction.

Condition (3). Under this condition $n=2(k-m)$, and so by our initial assumption $(m, k)>1$. Then $(n, m, k)=(2(k-m), m, k)=(m, k)>1$; but $(n, m, k)=1$ and so we have a contradiction.

Condition (4). Under this condition $n=2 k$, and so by our initial assumption $(m, k)>1$. Then $(n, m, k)=(2 k, m, k)=(m, k)>1$, but $(n, m, k)=1$ and so we have a contradiction.

\section{Abelianizations}

In this section we consider the abelianizations of groups $G_{n}(m, k)$. Using [13, Lemma 1.1] we have the following criterion that determines when $G_{n}(m, k)$ has infinite abelianization or when $G_{n}(m, k)$ is perfect. Let $f(t)=t^{m}-t^{k}+1$ and

$$
R_{n}(f)=\prod_{\theta^{n}=1} f(\theta)
$$

Then $G_{n}(m, k)^{\mathrm{ab}}$ is infinite if and only if $R_{n}(f)=0$, and $G_{n}(m, k)$ is perfect if and only if $R_{n}(f)= \pm 1$. 
Theorem 4. Suppose that $(n, m, k)=1$. Then $G_{n}(m, k)$ has infinite abelianization if and only if $n=0 \bmod 6$ and $(m \bmod 6, k \bmod 6)=(2,1)$ or $(4,5)$.

Proof. Observe that $R_{n}(f)=0$ if and only if $f(\lambda)=0$ for some $\lambda^{n}=1$.

Suppose first that $n=0 \bmod 6$ and $(\operatorname{mmod} 6, k \bmod 6)=(2,1)$ or $(4,5)$, and that $(n, m, k) \geqslant 1$. Let $\lambda^{3}=-1, \lambda \neq-1$; then $\lambda^{6}=1$, and therefore $\lambda^{n}=1$. If $m=2 \bmod 6$ and $k=1 \bmod 6$ then $f(\lambda)=\lambda^{2}-\lambda+1$. If $m=4 \bmod 6$ and $k=5 \bmod 6$ then $f(\lambda)=\lambda^{4}-\lambda^{5}+1=-\lambda+\lambda^{2}+1$. Thus $(\lambda+1) f(\lambda)=\lambda^{3}+1=0$ and since $(\lambda+1) \neq 0$ we have $f(\lambda)=0$.

For the converse, suppose that $(n, m, k)=1$ and that $f(\lambda)=0$ for some $\lambda^{n}=1$. Thus $|\lambda|=1$ so $\bar{\lambda}=\lambda^{-1}$ and then $f(\lambda)=0, f\left(\lambda^{-1}\right)=f(\bar{\lambda})=0$ imply

$$
\begin{gathered}
\lambda^{m}=\lambda^{k}-1, \\
\lambda^{-m}=\lambda^{-k}-1 .
\end{gathered}
$$

Hence $1=\lambda^{m} \cdot \lambda^{-m}=2-\lambda^{k}-\lambda^{-k}$, and so

$$
\lambda^{2 k}-\lambda^{k}+1=0 .
$$

Thus $\lambda^{k}=(1 \pm i \sqrt{3}) / 2$, and so $\lambda$ is a $(6 k)$ th root of unity. Since $\lambda^{n}=1,6 k$ divides $n$ and so, in particular,

$$
n=0 \bmod 6 .
$$

By (3.1) and (3.3) we have $\lambda^{m}=\lambda^{2 k}$, so that $\lambda^{m-2 k}=1$ and therefore

$$
m-2 k=0 \bmod 6 .
$$

Since $(n, m, k)=1$, it follows from $(3.4)$ and $(3.5)$ that $(m \bmod 6, k \bmod 6)=(2,1)$ or $(4,5)$, as required.

We remark that necessary and sufficient conditions for $G_{n}(m, k)$ to have infinite abelianization were incorrectly asserted in [17, Example 3(3)].

We now consider when $G_{n}(m, k)$ is perfect. By [1, Lemma 1.1], if $k=0$ or $m \bmod n$ then $G_{n}(m, k)$ is trivial, and hence perfect. Here is another sufficient condition:

Lemma 5. If $m=2 k \bmod n$ and $(n /(n, m, k), 6)=1$ then $G_{n}(m, k)$ is perfect.

Proof. Suppose first that $g(t)$ is any polynomial, and $d=(k, n)$. Then

$$
\begin{aligned}
\prod_{\theta^{n}=1} g\left(\theta^{k}\right) & =\prod_{q=0}^{n-1} g\left(e^{2 \pi i q k / n}\right)=\prod_{q=0}^{n-1} g\left(e^{2 \pi i q(k / d) /(n / d)}\right) \\
& =\left(\prod_{q=0}^{n / d-1} g\left(e^{2 \pi i q(k / d) /(n / d)}\right)\right)^{d}
\end{aligned}
$$


Since $(k / d, n / d)=1$, for each $q \in\{0, \ldots,(n / d-1)\}$ there exists a unique $Q \in\{0, \ldots,(n / d-1)\}$ such that $q(k / d)=Q \bmod n / d$. Hence

$$
\prod_{q=0}^{n / d-1} g\left(e^{2 \pi i q(k / d) /(n / d)}\right)=\prod_{Q=0}^{n / d-1} g\left(e^{2 \pi i Q /(n / d)}\right)=\prod_{\phi^{n / d}=1} g(\phi)
$$

and so

$$
\prod_{\theta^{n}=1} g\left(\theta^{k}\right)=\left(\prod_{\phi^{n /(n, k)}=1} g(\phi)\right)^{(n, k)}
$$

If $m=2 k \bmod n$ then setting $g(t)=t^{2}-t+1$ we have

$$
R_{n}(f)=\prod_{\theta^{n}=1} g\left(\theta^{k}\right)=\left(\prod_{\phi^{n /(n, k)}=1} g(\phi)\right)^{(n, k)}
$$

Now

$$
\begin{aligned}
\prod_{\phi^{n /(n, k)}=1} g(\phi) & =\prod_{\phi^{n /(n, k)}=1}\left(\phi-e^{2 \pi i / 6}\right) \prod_{\phi^{n /(n, k)}=1}\left(\phi-e^{-2 \pi i / 6}\right) \\
& =\left(\left(e^{2 \pi i / 6}\right)^{n /(n, k)}-1\right)\left(\left(e^{-2 \pi i / 6}\right)^{n /(n, k)}-1\right) \\
& =2-2 \cos \left(\frac{2 \pi n /(n, k)}{6}\right) \\
& =1
\end{aligned}
$$

since $(n /(n, k), 6)=(n /(n, m, k), 6)=1$. Hence $R_{n}(f)=1$ and $G_{n}(m, k)$ is perfect, as required.

Since this paper was written a complete description of the abelianization of $G_{n}(2 k, k)$ has been provided in [3, Lemma 7]. We conjecture that the sufficient conditions that we have given for $G_{n}(m, k)$ to be perfect are also necessary; we have verified this (using MaGMA) for $n \leqslant 200$.

Conjecture 6. If $G_{n}(m, k)$ is perfect then either $m=2 k \bmod n$ and $(n /(n, m, k), 6)=1$ or $k=0$ or $m \bmod n$.

Lemma 5 and Conjecture 6 form a natural generalization of [13, Theorem 2(ii), (iii)] (see also [11, Theorem 2.3(b)]). It seems likely that Odoni's methods [13] can be applied to prove Conjecture 6 in the general case. 


\section{Finiteness}

In [1, Question 1], Bardakov and Vesnin posed the following question:

Question. For which values of the defining parameters $n, m, k$ subject to the natural restrictions (1.2) are groups $G_{n}(m, k)$ finite?

If $(n, k)=1$ or $(n, m-k)=1$ then by [1, Lemma 1.3] the group $G_{n}(m, k)$ is isomorphic to some Gilbert-Howie group $H(n, t)$. More precisely, we have

Lemma 7. (i) If $(n, k)=1$ then

$$
G_{n}(m, k) \cong G_{n}(t, 1)=H(n, t)
$$

where $t k=m \bmod n$.

(ii) If $(n, k-m)=1$ then

$$
G_{n}(m, k) \cong G_{n}(t, 1)=H(t, 1)
$$

where $t(k-m)=n-m \bmod n$.

Proof. (i) See the proof of [1, Lemma 1.3].

(ii) By [1, Lemma 1.1(3)] we have

$$
G_{n}(m, k) \cong G_{n}(n-m, n-m+k)=G_{n}(K-k, K)
$$

where $K=n+(k-m)$. Then $(n, K)=(n, k-m)=1$, and so by (i) we have $G_{n}(K-k, K) \cong G_{n}(t, 1)$, where $t K=K-k \bmod n$, i.e. where

$$
t(k-m)=n-m \bmod n,
$$

as required.

Now the finite Gilbert-Howie groups $H(n, t)$ have almost been classified. In [11] the following theorem is proved:

Theorem 8 ([11]). Suppose that $(n, t) \neq(8,3),(9,3),(9,4),(9,6),(9,7)$. Then $H(n, t)$ is finite if and only if $t=0,1$ or $(n, t)=(2 k, k+1)$ where $k \geqslant 1$, or $(n, t) \in\{(3,2),(4,2),(5,2),(5,3),(5,4),(6,3),(7,4),(7,6)\}$.

As mentioned in Section 2, a calculation in MAGma shows that $H(8,3)$ is finite of order 295245. Cavicchioli, O'Brien and Spaggiari [3, Lemma 16] have recently proved that the (isomorphic) groups $H(9,3)$ and $H(9,6)$ are infinite. It remains unknown whether $H(9,4)$ and $H(9,7)$ define finite or infinite groups.

Suppose that $(n, k)>1$ and $(n, m-k)>1$, i.e. $G_{n}(m, k)$ is strongly irreducible. 
Since any non-trivial group with an aspherical presentation is infinite, the following is an immediate corollary of Theorem 2 and Lemma 3.

Corollary 9. Let $G=G_{n}(m, k)$ be strongly irreducible and assume that $G \neq 1$. Then $G$ is finite if and only if $(m, k)=1$ and $n=2 k$ or $n=2(k-m)$, in which case $G \cong \mathbb{Z}_{s}$ where $s=2^{n / 2}-(-1)^{m+n / 2}$.

Thus, to give a complete classification of the finite, strongly irreducible groups $G_{n}(m, k)$ it suffices to prove that every such (strongly irreducible) group is non-trivial. The problem as to which cyclically presented groups are trivial has been of interest recently; see for example [8], [9], [10], [12].

If Conjecture 6 holds, it follows that every strongly irreducible group $G_{n}(m, k)$ is not perfect, and hence non-trivial. To see this, observe that Conjecture 6 implies that if $G_{n}(m, k)$ is perfect then $m=2 k \bmod n$ and so $(n, m, k)=(n, k)$. But if $G_{n}(m, k)$ is strongly irreducible then $(n, m, k)=1,(n, k)>1$, a contradiction. Our MaGma calculations supporting Conjecture 6 therefore imply a classification of the finite, strongly irreducible groups $G_{n}(m, k)$ for $n \leqslant 200$.

Acknowledgement. Since this paper was originally written Eamonn O'Brien has pointed out that Theorem 4 is implied by an earlier result of Cavicchioli, Repovš and Spaggiari ([5, Corollary 5.8]). The author would like to thank Professor O'Brien for this and other helpful comments.

\section{References}

[1] V. G. Bardakov and A. Yu. Vesnin. A generalization of Fibonacci groups. Algebra and Logic 42 (2003), 131-160.

[2] W. Bosma, J. Cannon and C. Playoust. The Magma algebra system I: The user language. J. Symbolic Comput. 24 (1997), 235-265.

[3] A. Cavicchioli, E. A. O'Brien and F. Spaggiari. On some questions about a family of cyclically presented groups. (Preprint, 2007.)

[4] A. Cavicchioli, F. Hegenbarth and D. Repovš. On manifold spines and cyclic presentations of groups. In Knot theory, Banach Centre Publ. 42 (Polish Academy of Sciences, 1998), pp. 49-56.

[5] A. Cavicchioli, D. Repovš and F. Spaggiari. Topological properties of cyclically presented groups. J. Knot Theory Ramifications 12 (2003), 243-268.

[6] C. P. Chalk. Fibonacci groups with aspherical presentations. Comm. Algebra 26 (1998), 1511-1546.

[7] J. H. Conway. Advanced problem 5327. Amer. Math. Monthly 72 (1965), 915.

[8] M. Edjvet. On irreducible cyclic presentations. J. Group Theory 6 (2003), 261-270.

[9] M. Edjvet and P. Hammond. On a class of cyclically presented groups. Internat. J. Algebra Comput. 14 (2004), 213-240.

[10] M. Edjvet, P. Hammond and N. Thomas. Cyclic presentations of the trivial group. Experiment. Math. 10 (2001), 303-306.

[11] N. D. Gilbert and J. Howie. LOG groups and cyclically presented groups. J. Algebra $\mathbf{1 7 4}$ (1995), 118-131. 
[12] G. Havas and E. F. Robertson. Irreducible cyclic presentations of the trivial group. Experiment. Math. 12 (2003), 487-490.

[13] R. W. K. Odoni. Some Diophantine problems arising from the theory of cyclicallypresented groups. Glasgow Math. J. 41 (1999), 157-165.

[14] M. I. Prishchepov. Asphericity, atoricity and symmetrically presented groups. Comm. Algebra 23 (1995), 5095-5117.

[15] A. J. Sieradski. Combinatorial squashings, 3-manifolds, and the third homology of groups. Invent. Math. 84 (1986), 121-139.

[16] F. Spaggiari. Asphericity of symmetric presentations. Pub. Mat. 50 (2006), 133-147.

[17] A. Szczepański and A. Vesnin. HNN extension of cyclically presented groups. J. Knot Theory Ramifications 10 (2001), 1269-1279.

[18] R. M. Thomas. The Fibonacci groups revisited. In Groups, St Andrews 1989, vol. 2, London Math. Soc. Lecture Note Ser. 160 (Cambridge University Press, 1991), pp. 445-454.

[19] R. M. Thomas. On a question of Kim concerning certain group presentations. Bull. Korean Math. Soc. 28 (1991), 219-224.

Received 3 November, 2006

Gerald Williams, Department of Mathematical Sciences, University of Essex, Wivenhoe Park, Colchester CO4 3SQ, U.K.

E-mail: gwill@essex.ac.uk 